\title{
Hadron-Quark Crossover and Neutron Star Observations
}

Kota Masuda*†

Univ. of Tokyo / RIKEN

E-mail: masudaent.phys.s.u-tokyo.ac.ip

\section{Tetsuo Hatsuda}

RIKEN, Kavli IPMU

\section{Tatsuyuki Takatsuka}

RIKEN

On the basis of the percolation picture from the hadronic phase with hyperons to the quark phase with strangeness, we construct a new equation of state (EOS) with the pressure interpolated as a function of the baryon density. The maximum mass of neutron stars can exceed $2 M_{\odot}$ if the following two conditions are satisfied; (i) the crossover from the hadronic matter to the quark matter takes place at around three times the normal nuclear matter density, and (ii) the quark matter is strongly interacting in the crossover region and has stiff equation of state. This is in contrast to the conventional approach assuming the first order phase transition in which the EOS becomes always soft due to the presence of the quark matter at high density.

XV International Conference on Hadron Spectroscopy

4-8/11/2013

Nara, Japan

\footnotetext{
* Speaker.

${ }^{\dagger}$ These authors contributed equally to this work.
} 


\section{Introduction}

Any information from neutron stars (NSs) is quite useful to study the structure of high density matter. NSs have other observables such as mass $(M)$, radius $(R)$ the surface temperature $\left(T_{S}\right)$, the surface magnetic field $\left(B_{s}\right)$ and so on. Among others, $M$ and $R$ are particularly important probes for constraining the equation of state (EOS) and the composition of high density matter. For example, EOS of high density matter can be constrained from the mass-radius $(M-R)$ relationship of NSs through the Tolman-Oppenheimer-Volkov (TOV) equation. From the observational point of view, for NS binaries, typical value of the NS mass is about 1.4 solar mass. However, in 2010, a massive NS, PSR J1614-2230, with $M_{\mathrm{obs}}=(1.97 \pm 0.04) M_{\odot}$ was recently discovered by Shapiro delay technique [四]. And it was reported that a new massive NS, PSR J0348+0432 with $M_{\mathrm{obs}}=$ $(2.01 \pm 0.04) M_{\odot}$, was observed very recently[[]]. Motivated by this new observation, we would like to discuss two key questions in this paper; (i)how 2 solar mass NS can be sustained, and (ii) the fate of the quark matter inside such a heavy NS. The results of this paper was partially reported in [B], 田].

\section{Hadronic EOS (H-EOS)}

Before starting to discuss these questions in detail, let us first show the results of $M-R$ relationship with the conventional hadronic EOS (H-EOS). In Fig.W, we plot the $M-R$ relationship for various H-EOSs. Black line corresponds to APR EOS [[]]. APR uses AV18 two-body nuclear force, and phenomenological three-body force. But, there are no hyperons. Due to the effect of repulsive three-body force at high density, APR EOS can sustain 2 solar mass NS. However, the situation changes if we have hyperons at high density. The other lines have hyperons. Independent of the choice of the hadronic EOSs, huge softening due to hyperon mixture takes place. As a result, even the 1.44 solar mass neutron stars cannot be sustained with hyperon mixture. Upper (lower) red line, blue line and green line are TNI2u (TNI2), AV18+TBF and SCL3 $\Lambda \Sigma$ respectively.

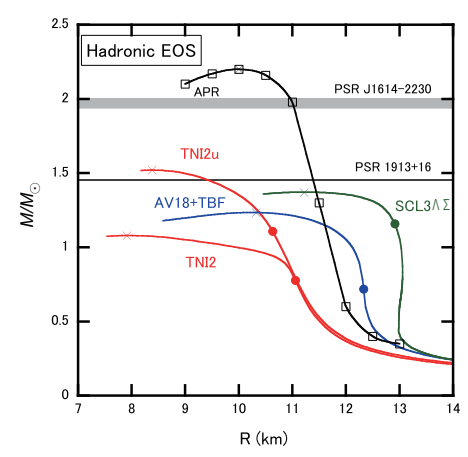

Figure 1: $M-R$ relationships with various H-EOSs. Solid black line: APR EOS [可]. Solid red lines: TNI2u (universal 3-body force with $\kappa=250 \mathrm{MeV}$ ) and TNI2 (3-nucleon force with $\kappa=250 \mathrm{MeV}$ ) [ [0, 四]. Solid blue line: AV18+TBF (G-matrix approach with hyperons) [8]]. Solid green line: SCL3 $\Lambda \Sigma$ (Relativistic mean field model with a chiral SU(3) symmetry)[国]. The gray band denotes $M=(1.97 \pm 0.04) M_{\odot}$ for PSR J1614-2230. The solid black line denotes $M=$ $1.44 M_{\odot}$ for PSR $1913+16$. 


\section{Hadron-Quark crossover}

In this section, let me discuss our idea of the hadron-quark crossover as a possible solution of the 2 solar mass puzzle. Our hadron-quark crossover is conceptually similar to the BEC-BCS crossover in ultracold atoms. As the inter-atomic attraction decreases, the system smoothly changes from the BEC state of dimers to the BCS state of Cooper pairs. Similar crossover may take place in high density QCD as shown by Fig.】. At low density, quarks are confined inside baryons. As the density increases, those finite size baryons start to overlap with each other. Eventually, the deconfined quark matter emerges at high density. The pressure as a function of density is a smooth curve. In the crossover region, baryons are overlapped and quarks are percolated. There is an important points related to this crossover scenario. H-EOS at low density and Quark EOS (we discuss later) at high density are merely the asymptotic behaviors. Since these EOSs are not trustable in the crossover region, there is no reason to justify the Gibbs criterion by using these EOSs. To model our percolation picture phenomenologically, I introduce a smooth interpolation of the pressure as a function of density by using a tanh function as this.

$$
P(\rho)=P_{H}(\rho) f_{-}(\rho)+P_{Q}(\rho) f_{+}(\rho), f_{ \pm}(\rho)=\frac{1}{2}\left(1 \pm \tanh \left(\frac{\rho-\bar{\rho}}{\Gamma}\right)\right),
$$

where $P_{H}$ and $P_{Q}$ are the pressure in the hadronic matter and that in the quark matter, respectively. To characterize the crossover region, we introduce two phenomenological parameters, $\bar{\rho}$ and $\Gamma . \bar{\rho}$ is the crossover density and $\Gamma$ is the width of the crossover region. We know that the normal nuclear matter is well described by hadronic EOS. So, we impose a condition that the crossover region is well separated from the normal nuclear matter. This is represented $\bar{\rho}>\rho_{0}+2 \Gamma$ in this paper. Once we have this pressure, the energy density can be derived by the thermodynamic relation.

\section{Quark EOS (Q-EOS)}

We consider (2+1)-flavor Nambu-Jona-Lasinio (NJL) model with 4-fermi and 6-fermi inter-

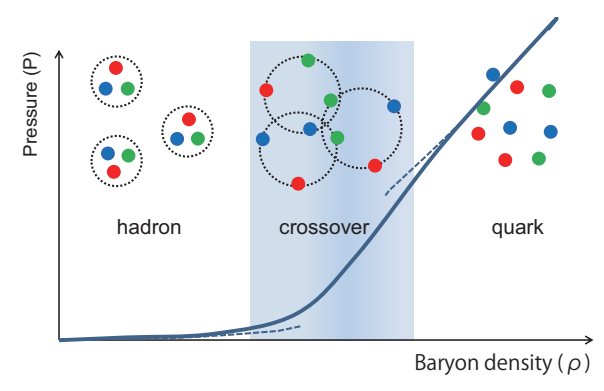

Figure 2: Schematic picture of the QCD pressure $(P)$ as a function of the baron density $(\rho)$ under the assumption of the hadron-quark crossover. The crossover region where finite-size hadrons start to overlap and percolate is shown by the shaded area. The pressure calculated on the basis of the point-like hadrons (shown by the dashed line at low density) and that calculated on the basis of weakly interacting quarks (shown by the dashed line at high density) lose their validity in the crossover region, so that the naive use of the Gibbs conditions by extrapolating the dashed lines is not justified in general. 
actions. This model is particularly useful to take into account the important phenomena such as the partial restoration of chiral symmetry at high density [ए]]. The model Lagrangian we consider is

$$
\mathscr{L}_{\mathrm{NJL}}=\bar{q}(i \theta-m) q+\frac{1}{2} G_{S} \sum_{a=0}^{8}\left[\left(\bar{q} \lambda^{a} q\right)^{2}+\left(\bar{q} i \gamma_{5} \lambda^{a} q\right)^{2}\right]-G_{D}\left[\operatorname{det} \bar{q}\left(1+\gamma_{5}\right) q+\text { h.c. }\right]-\frac{1}{2} g_{V}\left(\bar{q} \gamma^{\mu} q\right)^{2}
$$

where the quark field $q_{i}(i=u, d, s)$ has three colors and three flavors with the current quark mass $m_{i}$. The term proportional to $g_{V}(>0)$ gives a universal repulsion among different flavors. There are six independent parameters in the (2+1)-flavor NJL model; the UV cutoff, $\Lambda$, the coupling constants, $G_{S}, G_{D}$ and $g_{V}$, and the quark masses, $m_{u, d}$ and $m_{s}$. Most of the model parameters can be determined by hadron phenomenology in the vacuum. In this paper, we take the following parameter set (we call HK-parameter set [ए]]): $\Lambda=631.4 \mathrm{MeV}, G_{S} \Lambda^{2}=3.67, G_{D} \Lambda^{5}=9.29, m_{u, d}=5.5 \mathrm{MeV}, m_{s}=$ $135.7 \mathrm{MeV}$. However, there is one parameter which is not well determined phenomenologically. That is the vector-type 4-fermi interaction proportional to $g_{V}$. Since recent analysis [W], which is a comparison of the model and the lattice QCD data on the curvature of the critical line at zero chemical potential, suggests $g_{V}$ is about the same magnitude as the scalar interaction $G_{S}$, we consider three typical cases; $g_{V}=0, g_{V}=1.0 G_{S}$ and $g_{V}=1.5 G_{S}$. Then, we construct quark EOS in the mean field approximation with $\beta$-equilibrium and charge neutrality.

\section{Neutron star properties with $P$-interpolation}

In Fig. B(a), we show the $M-R$ relationship for various H-EOSs with hyperons whose onset is denoted by the filled circles. The crosses denote the points where maximum masses are realized: In all cases, the maximum mass $M_{\max }$ hardly exceeds 1.44 solar mass as we have discussed.

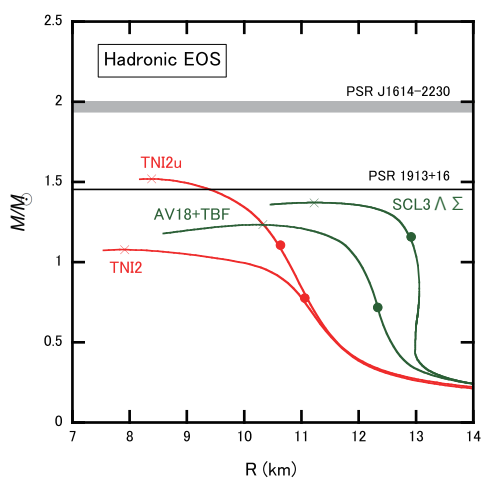

(a)

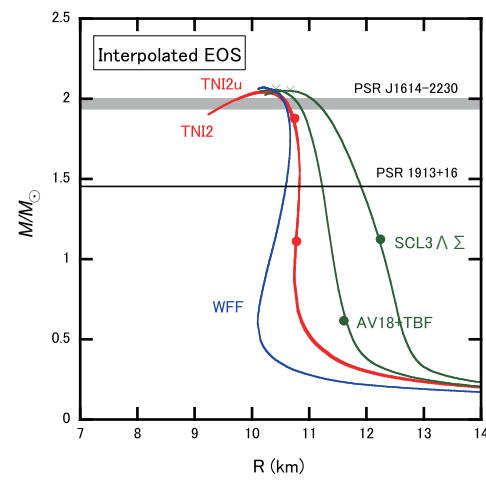

(b)

Figure 3: $\quad M-R$ relationships. (a) $M-R$ relationships with various H-EOS including hyperons. Solid red lines: TNI2u (universal 3-body force with $\kappa=250 \mathrm{MeV}$ ) and TNI2 (3-nucleon force with $\kappa=250 \mathrm{MeV}$ ). Solid green lines: AV18+TBF (G-matrix approach with hyperons) and SCL3 $\Lambda \Sigma$ (Relativistic mean field model with a chiral SU(3) symmetry). The gray band denotes $M=(1.97 \pm 0.04) M_{\odot}$ for PSR J1614-2230. The solid black line denotes $M=1.44 M_{\odot}$ for PSR 1913+16. (b) $M-R$ relationship with the EOS interpolated between H-EOS in (a) and Q-EOS with the HK parameter set and $g_{V}=G_{S}$, by the window parameters $(\bar{\rho}, \Gamma)=\left(3 \rho_{0}, \rho_{0}\right)$. Colors on each line are the same with those in (a). Solid blue line: WFF EOS [ㅁ]]. 
In Fig. B](b), we show the $M-R$ relationship with the EOS interpolated between H-EOS and QEOS. For the H-EOS, we consider the same EOSs as shown in Fig. B(a), while for the Q-EOS, we adopt the HK-parameter set with $g_{V}=G_{S}$ as a typical example. The crossover window is fixed to be $(\bar{\rho}, \Gamma)=\left(3 \rho_{0}, \rho_{0}\right)$. The red lines in Fig. B](b) correspond to the cases with TNI2u and TNI2, the green lines correspond to SCL3 $\Lambda \Sigma$ and AV18+TBF and the blue line correspond to WFF EOS [L2]. The onset of strangeness and the maximum mass are denoted by the filled circles and the crosses, respectively. Now, the maximum mass exceeds 2 solar mass, no matter what kind of hadronic EOS is taken. Maximum mass is essentially controlled by Quark EOS On the other hand, radius is essentially controlled by hadronic EOS.

\section{Summary and concluding remarks}

In this paper we have studied this problem on the basis of the percolation picture from the hadronic matter with hyperons to the quark matter with strange quarks. We have constructed an EOS by the interpolation between the H-EOS at lower densities and the Q-EOS at higher densities. We found that EOS can become stiffer due to the presence of quark matter, if the following conditions are met: (i)hadron-quark crossover occurs at relatively low densities, and (ii) quarks are strongly interacting at and above the crossover region. This conclusion is in contrast to the conventional EOS for hybrid stars derived through the Gibbs construction in which the resultant EOS becomes always softer than hadronic EOS and thereby leads to smaller $M_{\max }$. Our result implies that the observation of very massive NS cannot exclude the existence of the quark matter core. The quark matter can even increases the maximum mass of NSs. This is in contrast to the conventional belief, but is logically possible.

\section{References}

[1] P.B. Demorest et al., Nature 467, 1081 (2010)

[2] J. Antoniadis et al., Science 340, 6131 (2013).

[3] K. Masuda, T. Hatsuda and T. Takatsuka, Astrophys. J. 794, 12 (2013).

[4] K. Masuda, T. Hatsuda and T. Takatsuka, Prog. Theor. Exp. Phys. 073Do1 (2013).

[5] A. Akmal, V.R. Pandharipande and D.G. Ravenhall, Phys. Rev. C 58, 1804 (1998)

[6] S. Nishizaki, Y. Yamamoto and T. Takatsuka, Prog. Theor. Phys. 105, 607 (2001)

[7] S. Nishizaki, Y. Yamamoto and T. Takatsuka, Prog. Theor. Phys. 108, 703 (2002)

[8] M. Baldo, G.F. Burgio and H.J. Schulze, Phys. Rev. C 61, 055801 (2000)

[9] K. Tsubakihara, H. Maekawa, H. Matsumiya and A. Ohnishi, Phys. Rev. C 81, 065206 (2010)

[10] T. Hatsuda and T. Kunihiro, Phys. Rep. 247, 221 (1994)

[11] N. M. Bratovic, T. Hatsuda and W. Weise, Phys. Lett. B 719, 131 (2013)

[12] B. Friedman and V. R. Pandharipande, Nucl. Phys. A 361, 502 (1981) 